\title{
A sazonalidade do tromboembolismo venoso no clima subtropical de São Paulo
}

\author{
Seasonal variation of venous thromboembolism in the subtropical \\ climate of São Paulo, Brazil
}

\section{Daniela Kleinfelder ${ }^{1}$, Jó Luis Andrade ${ }^{2}$, Sascha Werner Schlaad ${ }^{3}$, Francine Correa Carvalho ${ }^{4}$, Bonno van Bellen ${ }^{5}$}

\section{Resumo}

Contexto: Os fatores desencadeantes da doença tromboembólica venosa vêm sendo cada vez melhor identificados. Causas externas podem influir na sua ocorrência, e algum destaque tem sido dado a fatores climáticos. Nada se sabe quanto a essa interferência em nossa latitude.

Objetivos: Analisar se há diferença na incidência do tromboembolismo venoso de acordo com as estações do ano, num hospital da cidade de São Paulo, Brasil, cujo clima é categorizado como subtropical.

Métodos: Foi realizado trabalho retrospectivo de levantamento de dados a partir de prontuários de pacientes cujo diagnóstico de internação ou óbito foi de trombose venosa profunda ou tromboembolismo pulmonar, no período de janeiro de 1996 a outubro de 2003, no Hospital da Beneficência Portuguesa de São Paulo. Para comparação e estudo, os casos foram agrupados em trimestres (primeiro trimestre $=$ janeiro, fevereiro e março; segundo trimestre $=$ abril, maio e junho; terceiro trimestre $=$ julho, agosto e setembro; e quarto trimestre $=$ outubro, novembro e dezembro) e conforme sua ocorrência nos chamados meses quentes e frios, de acordo com a média de temperatura mensal (meses quentes = outubro a abril; meses frios = maio a setembro) .

Resultados: Foram encontrados 955 casos de tromboembolismo venoso no período analisado. Foi utilizado o teste ANOVA para análise, que não revelou diferença estatisticamente significativa na incidência do tromboembolismo venoso de acordo com os trimestres. Quando analisados separadamente, também não se evidenciou significância estatística em relação ao tromboembolismo pulmonar e à trombose venosa profunda. Quando comparados os meses quentes e frios, observou-se aumento da incidência de trombose venosa profunda nos meses quentes ( $\mathrm{p}<0,05$, teste de Mann-Whitney).

Conclusão: $\mathrm{O}$ tromboembolismo venoso é uma doença que não tem uma relação bem estabelecida com as variações climáticas. A influência da temperatura ambiental na coagulabilidade ainda precisa ser amplamente estudada.

Palavras-chave: Trombose venosa, embolia pulmonar, epidemiologia, climatologia.

\begin{abstract}
Background: The triggering factors of venous thromboembolic disease have been increasingly clarified. External causes may influence its occurrence, and some climactic factors have stood out. Nothing is known about such interference in our latitude.
\end{abstract}

Objectives: To determine whether there are seasonal variations in venous thromboembolism in a hospital-based population in São Paulo, Brazil, which has subtropical climate.

Methods: Medical records of patients admitted to Hospital da Beneficência Portuguesa de São Paulo with the diagnosis of deep venous thrombosis or pulmonary thromboembolism were reviewed from January 1996 to October 2003. Cases were grouped in trimesters (first trimester = January, February and March; second trimester $=$ April, May and June; third trimester $=$ July, August and September; fourth trimester $=$ October, November and December $)$. They were also grouped as to warm and cold months, according to mean temperature (warm months = October through April; cold months = May through September .

Results: A total of 955 cases of venous thromboembolism were found during the study period. The ANOVA test was used for statistical analysis, showing no significant difference in the occurrence of venous thromboembolism considering the four trimesters. Separate analysis of deep venous thrombosis and pulmonary embolism incidence showed no differences either. Comparing warm and cold months, there was an increased incidence of deep venous thrombosis during warm months ( $\mathrm{p}<0.05$, Mann-Whitney test).

Conclusion: Venous thromboembolism is not clearly related to climatic variations. The influence of climate and temperature on blood coagulability is poorly understood and needs to be further studied.

Keywords: Venous thrombosis, pulmonary embolism, epidemiology, climatology.

1. Curso de Pós-Graduação Lato Sensu em Cirurgia Vascular e Angiologia, Hospital Beneficência Portuguesa de São Paulo (BPSP), São Paulo, SP.

2. Curso de Pós-Graduação Lato Sensu em Cirurgia Vascular Integrada, BPSP, São Paulo, SP. Professor, Cirurgia Geral, Faculdade de Medicina, Universidade Estadual do Sudoeste da Bahia (UESB), Vitória da Conquista, BA.

3. Curso de Pós-Graduação Lato Sensu em Cirurgia Vascular Integrada, BPSP, São Paulo, SP.

4. Doutora em Cardiologia, Instituto do Coração, Faculdade de Medicina da Universidade de São Paulo (InCOR-FMUSP), São Paulo, SP. Cardiologista, Serviço de Cirurgia Vascular e Angiologia, BPSP, São Paulo, SP.

5. Livre-docente, Moléstias Vasculares Periféricas, Universidade Estadual de Campinas (UNICAMP), Campinas, SP. Chefe, Serviço de Cirurgia Vascular e Angiologia, BPSP, São Paulo, SP. Coordenador, Curso de Pós-Graduação Lato Sensu em Cirurgia Vascular Integrada, BPSP, São Paulo, SP.

Trabalho realizado no Serviço de Cirurgia Vascular e Angiologia do Hospital São Joaquim, Real e Benemérita Sociedade Portuguesa de Beneficência de São Paulo, SP.

Não foram declarados conflitos de interesse associados à publicação deste artigo.

Artigo submetido em 07.03.08, aceito em 26.11.08.

J Vasc Bras. 2009;8(1):29-32.

Copyright $\odot 2009$ by Sociedade Brasileira de Angiologia e de Cirurgia Vascular 


\section{Introdução}

O tromboembolismo venoso (TEV) é uma importante causa de morbimortalidade nos países ocidentais ${ }^{1}$. Somente nos EUA são relatados mais de 100 casos $/ 100.000$ pessoas/ano ${ }^{2}$. No Brasil e nos demais países da América Latina, são escassos os trabalhos publicados sobre a epidemiologia do $\mathrm{TEV}^{3}$. Maffei et al. estimaram incidência de 0,6 casos de trombose venosa profunda (TVP) para cada 1.000 habitantes/ano, na cidade de Botucatu ${ }^{4}$. Amari et al. verificaram incidência de $6 \%$ de tromboembolismo pulmonar (TEP), em estudo de necropsia, na Santa Casa de São Paulo ${ }^{5}$.

A média de idade populacional tem aumentado, e também o número absoluto de casos de TEV ${ }^{6}$. Parte desses casos se manifestará como TEP. Na Inglaterra, o TEP é causa de 200.000 óbitos/ano, correspondendo a 1/10 das mortes intra-hospitalares ${ }^{7}$, e é a causa de morte evitável mais comum em pacientes internados nos EUA ${ }^{8}$. Do total de casos de TEP, $10 \%$ são fatais em menos de 1 hora do início do evento ${ }^{9}$, e aproximadamente $5 \%$ dos pacientes com TEP desenvolvem hipertensão pulmonar ${ }^{9}$. A síndrome de estase venosa crônica atinge $11 \%$ dos pacientes com TEV, evoluindo para úlceras varicosas em 3,7 $\pm 0,9 \%$ dos pacientes em 20 anos $^{9}$.

Muito tem sido feito quanto ao desenvolvimento de métodos e medicamentos para a profilaxia do TEV e, consequentemente, muito se tem evoluído no que tange à compreensão das causas e aos fenômenos epidemiológicos da doença.

Vários estudos sugerem variação da incidência do tromboembolismo venoso de acordo com as estações climáticas ${ }^{2,7,10-16}$. No entanto, esses trabalhos foram realizados em países de climas temperados. Nenhum estudo foi encontrado originado em país tropical.

O objetivo deste trabalho é analisar se há diferença na incidência do TEV de acordo com as estações do ano, num hospital da cidade de São Paulo, situada no Trópico de Capricórnio, limite entre o clima tropical e temperado.

\section{Casuística e métodos}

Foi feito levantamento retrospectivo de dados a partir de prontuários de pacientes cujo diagnóstico de internação ou óbito foi de TVP ou TEP, no período de janeiro de 1996 a outubro de 2003, no Hospital da Beneficência Portuguesa de São Paulo.

Para comparação e estudo, os casos foram agrupados em trimestres:

- Primeiro trimestre: janeiro, fevereiro e março;

- Segundo trimestre: abril, maio e junho;

- Terceiro trimestre: julho, agosto e setembro;

- Quarto trimestre: outubro, novembro e dezembro.

Foi utilizado o teste ANOVA para análise.

$\mathrm{O}$ ano também foi dividido em meses quentes e meses frios. Essa caracterização foi feita considerando-se a média de temperatura mensal $\geq 20^{\circ} \mathrm{Ce}<20^{\circ} \mathrm{C}$, respectivamente. Os meses quentes são os compreendidos entre outubro e abril, sendo que os meses frios são os de maio a setembro. Os dados foram obtidos a partir de informações do site The Weather Channel ${ }^{17}$. Foi utilizado o teste de Mann-Whitney para análise estatística.

Foi considerado haver significância estatística quando $\mathrm{p}<0,05$.

\section{Resultados}

Foram encontrados 955 casos de TEV, sendo 453 casos de TVP e 502 casos de TEP (média de 10,2 casos de TEV por mês, com desvio padrão de 4,9).

No primeiro trimestre, foram encontrados 253 casos de TEV (26,5\%), sendo 143 casos de TVP (31,6\%) e 110 casos de TEP $(21,9 \%)$. No segundo trimestre, foram diagnosticados 232 casos de TEV (24,3\%), correspondendo a 100 casos de TVP $(22,1 \%)$ e 132 casos de TEP $(26,3 \%)$. No terceiro trimestre, identificou-se 223 casos de TEV (23,3\%), com 94 casos de TVP (20,7\%) e 129 casos de TEP $(25,7 \%)$. No quarto trimestre, obteve-se 247 casos de TEV (25,9\%), sendo 116 casos de TVP $(25,6 \%)$ e 131 casos de TEP $(26,1 \%)$.

O teste ANOVA revelou não haver diferença estatisticamente significativa na incidência do TEV de acordo com os trimestres. Quando analisados separadamente, também não evidenciou significância estatística em relação ao TEP e à TVP.

Quanto aos meses quentes (de outubro a abril, temperatura média $>20^{\circ} \mathrm{C}$ ) e meses frios (maio a setembro, temperatura média $<20^{\circ} \mathrm{C}$ ), houve, nos meses quentes, 


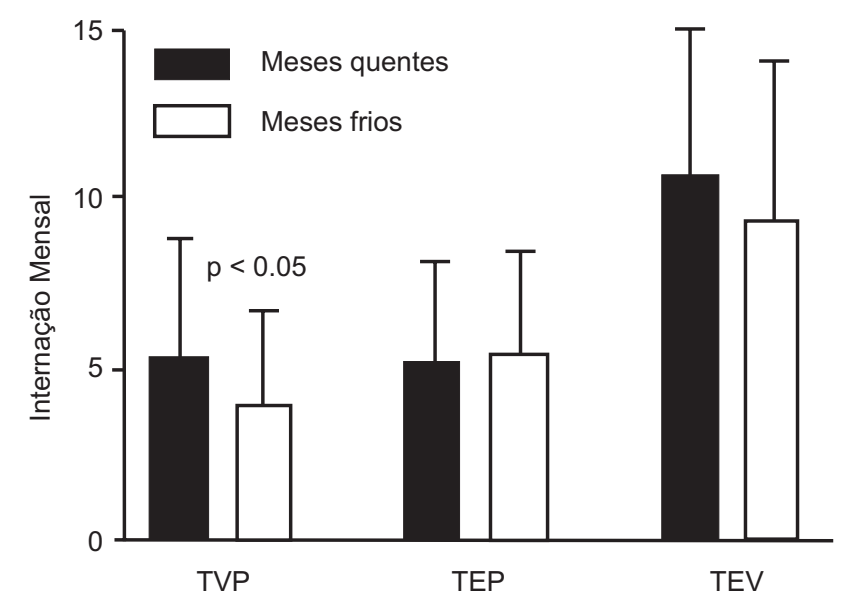

$\mathrm{TEV}=$ tromboembolismo venoso $\mathrm{TEP}=$ tromboembolismo pulmonar; $\mathrm{TVP}=$ trombose venosa profunda.

Figura 1 - Distribuição das médias de internação mensal por TVP, TEP e TEV, durante os meses quentes e frios (médias e desvio padrão)

578 casos de TEV (60,5\%), sendo 283 casos de TEP $(56,4 \%)$ e 295 casos de TVP $(65,1 \%)$. Nos meses frios, ocorreram 377 casos de TEV (39,5\%), sendo 219 casos de TEP $(43,6 \%)$ e 158 casos de TVP $(34,8 \%)$. O teste de Mann-Whitney mostrou aumento dos casos de TVP nos meses quentes $(\mathrm{p}<0,05)$ (Figura 1).

\section{Discussão}

Diversos estudos relataram maior incidência de morte e admissões hospitalares por doença coronariana e acidente vascular cerebral no inverno ${ }^{10,18}$. Da mesma forma, alguns trabalhos demonstraram maior incidência de TEP fatal no inverno do que no verão $0^{2,7,11,18}$, bem documentada em metanálise de 23 trabalhos envolvendo 11.000 casos $^{12,13}$. Num estudo inglês de necropsia, observou-se maior incidência de embolismo pulmonar no outono e na primavera ${ }^{14}$.

Bounameaux et al. ${ }^{10}$, em 1996, não encontraram variação sazonal na incidência de TVP, num estudo retrospectivo realizado com 9.208 pacientes, no período de 1989 a 1994, em Genebra, cidade com grandes diferenças de temperatura entre verão e inverno, assim como o estudo francês de Galle et al., realizado em $1998^{15}$, e o estudo americano de Stein et al. ${ }^{16}$. Este avaliou variações sazonais do TEV, num período de 21 anos, tanto em regiões com grandes variações de temperatura quanto em áreas com pouca diferença climática entre as estações do ano.

No entanto, Boulay et al. ${ }^{13}$, em 2001, publicaram um estudo realizado na França, incluindo 62.237 pacientes, mostrando a existência de variação sazonal na ocorrência de TEV, com número de admissões hospitalares $18 \mathrm{e}$ $22 \%$ acima da média nos meses frios para TVP e para TEP, respectivamente.

As prováveis razões para a variação sazonal também foram discutidas nos diversos trabalhos publicados acerca do assunto. White considera que a diminuição da atividade física no inverno possa apresentar relação com o desenvolvimento da TVP 2 . Boulay et al. ${ }^{13}$ hipotetizam que a vasoconstrição induzida pelo frio e a redução da atividade física produzem uma diminuição bem documentada no fluxo sanguíneo dos membros inferiores; e, ademais, as infecções do trato respiratório do inverno podem induzir à hipercoagulabilidade. Outros autores acreditam que a alteração da temperatura é um fator indireto e que a diminuição da duração do dia e, portanto, da luz solar interfere na produção de melatonina e na coagulabilidade ${ }^{12}$. Wilmshurst ${ }^{18}$ ainda acrescenta que a concentração plasmática de fibrinogênio é inversamente relacionada à temperatura ambiente, sendo que parte desse aumento do fibrinogênio no inverno pode ser resultante das infecções respiratórias sazonais. Em condições frias, alguns fatores de coagulação estão aumentados in vitro, como a contagem de plaquetas e a agregação plaquetária. A diminuição do volume plasmático e o aumento da viscosidade do sangue durante a exposição ao frio tendem a promover trombose. Entretanto, muito pouco continua sendo conhecido sobre a flutuação sazonal da coagulabilidade ${ }^{13}$.

Em nosso estudo, não encontramos diferença de incidência para os casos de TEV quando consideramos os trimestres, que corresponderiam às estações climáticas. Porém, foi observado aumento na incidência de TVP nos meses quentes. A determinação de meses quentes e frios foi baseada na média de temperatura dos 12 meses, que é de $20^{\circ} \mathrm{C}^{17}$, e por isso foi usada como divisor. Esse valor não apareceu em outros trabalhos, pois foram realizados em países europeus ou nos EUA, onde as variações de temperatura são mais marcantes, e não 
conseguimos equiparar com o clima brasileiro para definir o mesmo valor de "corte" para a temperatura. $\mathrm{Na}$ ocasião do estudo, não encontramos nenhum trabalho brasileiro ou de países com clima semelhante para fins de análise comparativa.

Não foi encontrada nenhuma teoria na literatura que explicasse o aumento da incidência de TVP nos meses mais quentes. Mais difícil ainda é explicar o porquê da ausência de correlação entre a incidência de TVP e o TEP, já que se poderia esperar um paralelismo entre os dois fenômenos. Da mesma forma que se encontra dificuldade para explicar o aumento do TEV nos meses frios do hemisfério norte, poder-se-ia especular que o calor promove maior desidratação e mantém as pessoas em estado mais sedentário, o que, por sua vez, poderia promover aumento da incidência de TVP.

Pouco ainda é conhecido sobre a flutuação sazonal na coagulabilidade, e outras variáveis deveriam ser analisadas conjuntamente para afastar a possibilidade de a temperatura ser apenas um fator confusional nesses estudos.

\section{Conclusão}

O TEV é uma doença que não tem relação bem estabelecida com as variações climáticas. A influência da temperatura ambiental na coagulabilidade ainda precisa ser amplamente estudada.

\section{Referências}

1. Arcelus JI, Caprini JA, Monreal M, Suárez C, González-Fajardo J. The management and outcome of acute venous thromboembolism: a prospective registry including 4011 patients. J Vasc Surg. 2003;38:916-22.

2. White RH. The epidemiology of venous thromboembolism. Circulation. 2003;107(23 Suppl 1):I4-8.

3. Silva MC. Tromboembolismo venoso: epidemiologia e fatores de risco. In: Brito CJ, Duque A, Merlo I, Murilo R, Fonseca VL, editores. Cirurgia vascular. Rio de Janeiro: Revinter; 2002. p. 1123-34.

4. Maffei FHA. Epidemiologia da trombose venosa profunda e de suas complicações no Brasil. Cir Vasc Angiol 1998;14:5-8.
5. Amary J, Coli Jr DF, Pereira M, Bailone S. Embolismo pulmonar - levantamento em 13500 necrópsias. Arq Hosp Santa Casa S Paulo. 1974;20:143-7.

6. Heit JA. Venous thromboembolism epidemiology: implications for prevention and management. Semin Thromb Hemost. 2002;28 Suppl 2:3-13.

7. Cooke EA, McNally MA, Mollan RAB. Seasonal variations in fatal pulmonary embolism. Several mechanisms contribute. BMJ. 1995;310:129.

8. Anderson FA, Spencer FA. Risk factors for venous thromboembolism. Circulation. 2003;107(23 Suppl 1):19-16.

9. Kearon C. Natural history of venous thromboembolism. Circulation. 2003;107(23 Suppl 1):I22-30.

10. Bounameaux H, Hicklin L, Desmarais S. Seasonal variation in deep vein thrombosis. BMJ. 1996;312:284-5.

11. Gallerani M, Manfredini R, Ricci L, et al. Sudden death from pulmonary thromboembolism: chronobiological aspects. Eur Heart J. 1992;13:661-5.

12. Allan TM, Douglas AS. Seasonal variation in deep vein thrombosis. Fatal pulmonary embolism is increased in both autumn and winter. BMJ. 1996;312:1227.

13. Boulay F, Berthier F, Schoukroun G, Raybaut C, Gendreike Y, Blaive B. Seasonal variations in hospital admission for deep vein thrombosis and pulmonary embolism: analysis of discharge data. BMJ. 2001;323:601-2.

14. Green J, Edwards C. Seasonal variation in the necropsy incidence of massive pulmonary embolism. J Clin Pathol. 1994;47:58-60.

15. Galle C, Wautrecht JC, Motte S, et al. Rôle de la saison dans l'incidence de la thrombose veineuse profonde. J Mal Vasc. 1998;23:99-101.

16. Stein PD, Kayali F, Olson RE. Analysis of ocurrence of venous thromboembolic disease in the four seasons. Am J Cardiol. 2004;93:511-3.

17. The Weather Channel [site na Internet]. The Weather Channel Interactive, Inc. Acess: 01/04. http://br.weather.com/weather/ climatology/BRXX0232

18. Wilmshurst P. Temperature and cardiovascular mortality. BMJ. 1994;309:1029-30.

Correspondência:

Dr. Bonno van Bellen

Rua Maestro Cardim, 925, Liberdade

01323-001 - São Paulo, SP

Tel.: (11) 3283.2955

E-mail: bellen@apm.org.br 